\title{
Guiding the visually impaired through the environment with beacons
}

\author{
Author(s) \\ van der Bie, Joey; Visser, Britte; Matsari, Jordy; Singh, Mijnisha; Van Hasselt, Timon; \\ Koopman, Jan; Kröse, Ben \\ DOI \\ 10.1145/2968219.2971387
}

Publication date

2016

\section{Published in}

Proceedings of the 2016 ACM International Joint Conference on Pervasive and Ubiquitous Computing

Link to publication

\section{Citation for published version (APA):}

van der Bie, J., Visser, B., Matsari, J., Singh, M., Van Hasselt, T., Koopman, J., \& Kröse, B. (2016). Guiding the visually impaired through the environment with beacons. In P. Lukowicz (Ed.), Proceedings of the 2016 ACM International Joint Conference on Pervasive and Ubiquitous Computing: Adjunct (pp. 385-388). Association for Computing Machinery. https://doi.org/10.1145/2968219.2971387

If you believe that digital publication of certain material infringes any of your rights or (privacy) interests, please let the Library know, stating your reasons. In case of a legitimate complaint, the Library will make the material inaccessible and/or remove it from the website. Please contact the library:

https://www.amsterdamuas.com/library/contact/questions, or send a letter to: University Library (Library of the University of Amsterdam and Amsterdam University of Applied Sciences), Secretariat, Singel 425, 1012 WP Amsterdam, The Netherlands. You will be contacted as soon as possible.

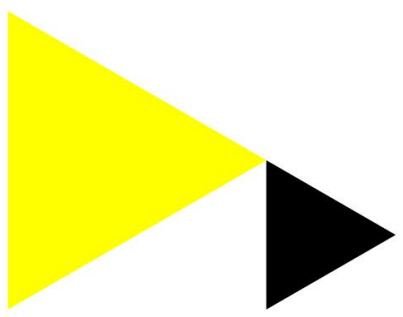


See discussions, stats, and author profiles for this publication at: https://www.researchgate.net/publication/307444641

\section{Guiding the Visually Impaired through the Environment with Beacons}

Conference Paper · September 2016

CITATIONS

0
READS

10

All content following this page was uploaded by Joey van der Bie on 30 August 2016.

The user has requested enhancement of the downloaded file. All in-text references underlined in blue are added to the original document and are linked to publications on ResearchGate, letting you access and read them immediately. 


\section{Guiding the Visually Impaired through the Environment with Beacons}

\author{
Joey van der Bie \\ Britte Visser \\ Jordy Matsari \\ Mijnisha Singh \\ Digital Life Centre \\ Amsterdam University of \\ Applied Sciences \\ Amsterdam, The Netherlands \\ j.h.f.van.der.bie@hva.nt \\ britte.visser@hva.nl \\ jordy.matsari@hva.nl \\ mijnisha.singh@hva.nl \\ Timon van Hasselt \\ Jan Koopman
Royal Dutch Visio
Centre of expertise for visually
impaired people
Amsterdam, The Netherlands
timonvanhasselt@visio.org
jankoopman@visio.org \\ Jan Koopman
Royal Dutch Visio
Centre of expertise for visually
impaired people
Amsterdam, The Netherlands
timonvanhasselt@visio.org
jankoopman@visio.org \\ Jan Koopman
Royal Dutch Visio
Centre of expertise for visually
impaired people
Amsterdam, The Netherlands
timonvanhasselt@visio.org
jankoopman@visio.org \\ Jan Koopman
Royal Dutch Visio
Centre of expertise for visually
impaired people
Amsterdam, The Netherlands
timonvanhasselt@visio.org
jankoopman@visio.org \\ Jan Koopman
Royal Dutch Visio
Centre of expertise for visually
impaired people
Amsterdam, The Netherlands
timonvanhasselt@visio.org
jankoopman@visio.org \\ Jan Koopman
Royal Dutch Visio
Centre of expertise for visually
impaired people
Amsterdam, The Netherlands
timonvanhasselt@visio.org
jankoopman@visio.org \\ Jan Koopman
Royal Dutch Visio
Centre of expertise for visually
impaired people
Amsterdam, The Netherlands
timonvanhasselt@visio.org
jankoopman@visio.org

\section{Ben Kröse} \\ University of Amsterdam \\ Amsterdam, The Netherlands \\ b.j.a.krose@uva.nl \\ Digital Life Centre \\ Amsterdam University of \\ Applied Sciences \\ Amsterdam, The Netherlands \\ b.j.a.krose@hva.nl
}

Permission to make digital or hard copies of part or all of this work for personal or classroom use is granted without fee provided that copies are not made or distributed for profit or commercial advantage and that copies bear this notice and the full citation on the first page. Copyrights for third-party components of this work must be honored. For allights or third-party components of this work must be honored. For all other uses, contact the Owner/Author.

Copyright is held by the owner/author(s).

Ubicomp/ISWC'16 Adjunct, September 12-16, 2016, Heidelberg,

Germany

ACM 978-1-4503-4462-3/16/09.

http://dx.doi.org/10.1145/2968219.2971387.

\begin{abstract}
Urban environments are full of noise and obstacles, and therefore potentially dangerous and difficult to navigate for the visually impaired. Using Bluetooth beacons and a smartphone app we guide them through these environments by providing the information needed for that specific location. We present the preliminary results concerning the usability of our approach.
\end{abstract}

\section{Author Keywords}

Bluetooth; beacon; smartphone; visually impaired; smart city; navigation;

\section{ACM Classification Keywords}

H.5.2. Information interfaces and presentation (e.g., $\mathrm{HCI}$ ): User Interfaces; Interaction styles

\section{Introduction}

Visually impaired people experience trouble with navigation and orientation due to their weakened ability to rely on eyesight to monitor the environment [1][2]. Smartphones such as the iPhone are already popular devices among the visually impaired for navigating [3]. Could this device also be helpful for orientating? We developed an iPhone application that responds to Bluetooth beacons to inform the user about their environment. We tested the implementation in an urban environment with visually impaired people using 
the route from the Amsterdam Bijlmer train station to an office of Royal Dutch Visio.

\section{Related Work}

Verbal descriptions of local geometric detail improve wayfinding and accuracy [4]. In addition to the current navigational methods, the visually impaired would like to see information about their environment such as lampposts, trees and other obstacles in the public space [2]. Atkins et al. showed that beacons can be used to supply ad hoc information about a nearby obstacle in the environment where GPS cannot [5]. Havik et al. developed a protocol for instructions supplied by RFID beacons [6], dictating the supply of detailed information about the environment, important landmarks and information about the next navigation point.

\section{Method}

We tested if we could aid the navigation of visually impaired through an urban environment using iBeacons and a smartphone application. Bluetooth beacons were attached at two meters high to lampposts and traffic signs along a specified route to give the user instructions via a custom made iPhone app. Three different obstacle types were identified and implemented in the app: a crossover with traffic signs, a car parking entrance and objects blocking the pathway like stairs. Following the protocol of Havik et al. [6] at each obstacle the beacon will trigger the app to present important information about the surroundings like potential hazards nearby, how to navigate around or through obstacles and information about the next obstacle. The information is presented using pictures of the environment and instructions in text. The application uses Apple's accessibility features to communicate the instructions with VoiceOver screenreader [4]. Furthermore the app allows the user to preview the route, to prepare for upcoming obstacles and landmarks. Last, users can customize the app by specifying the amount of detail in images and information the app presents.

To determine if the app is more useful for the participants than their current navigational method, participants walked the route both with and without the application. When walking with the app, participants were guided by the app. When walking without the app they used their own navigational method. During both walks a supervisor ensured the safety of the participant.

During both walks, after each obstacle, participants were asked how safe they felt. We used a five point Likert scale where one stood for "feeling very safe" and five for "feeling very unsafe".

Qualitative feedback on the usability of the app was collected via two methods: 1 . During the test While walking the route participants were asked to speak their thoughts about the app. 2. After having walked the route the participants were asked several questions about the application. 


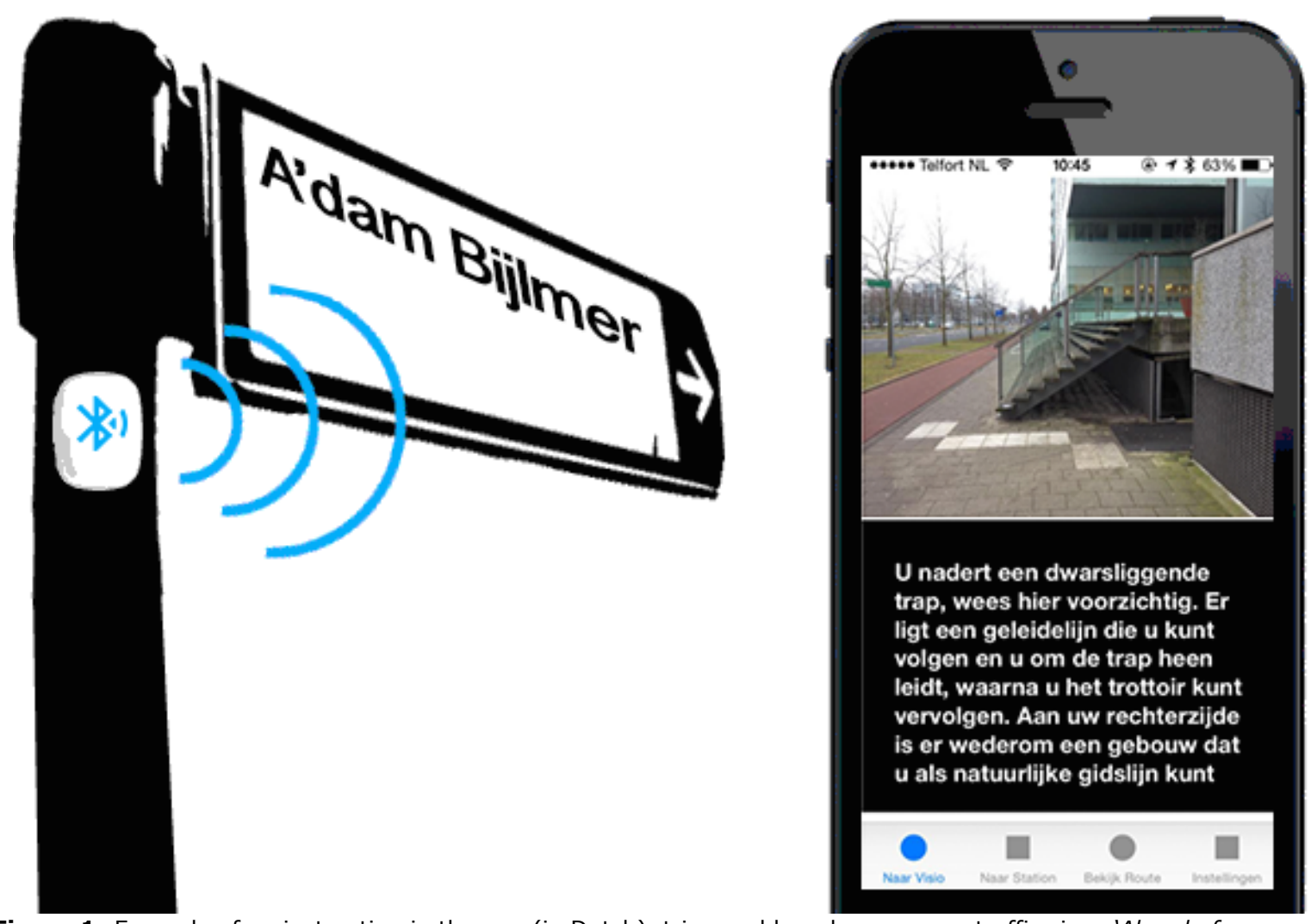

Figure 1: Example of an instruction in the app (in Dutch), triggered by a beacon on a traffic sign: 'Ahead of you are stairs that partially block the path. A guideline will direct you around the stairs, after which you will be able to continue following the sidewalk. On your right there will be a building that you can follow as a guide line.'

\section{Results}

Five visually impaired participated, one female and five males, age range from 30 to 78 and with varying levels of visual limitations. Three participants were familiar with the route and two walked the route for the first time.

After each obstacle participants rated how safe they felt on a five point Likert scale. We normalized the results by deducting the scores of the walk without the app from the scores of the walk with the app. The average of all participants is shown in figure 2. When passing the traffic light halfway during the route we see that the participants feel safer with than without the app.

Summarizing the qualitative feedback, we noticed that all participants indicated feeling supported by the app. They found the type of instructions ideal for walking and learning new routes. Of the five participants, three found the length of the instructions appropriate and two found them too long. They would like to split the detailed instructions in a short instruction and the 


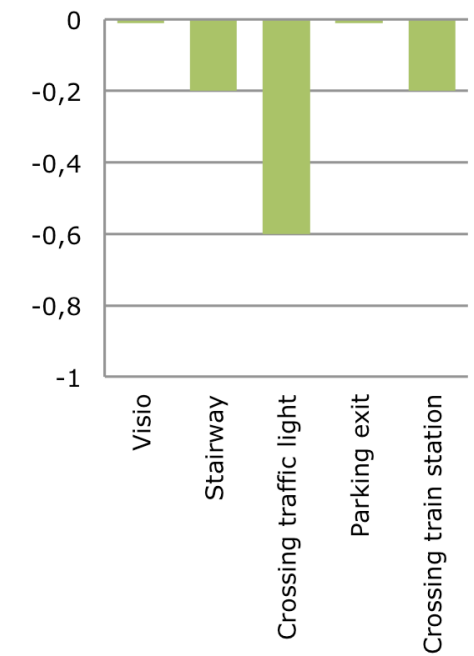

Figure 2: Visualization of normalized safety scores per obstacle. The lower the score, the more secure they felt while walking with the app. option for more detailed instructions. They felt that a detailed instruction gave too much information in a hazardous environment like a crossover. Two participants found the information focused on orientation not necessary, while three participants liked knowing their surroundings.

\section{Conclusion and discussion}

We developed a prototype app that gives users instructions when they are in the proximity of a beacon. These instructions inform about potential hazards ahead of them and about nearby recognizable landmarks. We tested our app with five visual impaired people along the route from the Amsterdam Bijlmer train station to an office of Royal Dutch Visio to determine if their sense of security improved by using the application.

Looking at the safety questions we see that participants felt safer with the app, especially when crossing the road with traffic lights. We believe this big difference in comparison to the other obstacles is due to the crossover being considered more dangerous than the other obstacles. This is reflected by their feedback in requesting less direct information at these locations.

All participants indicated feeling supported and at ease with our application, stating they would use the application when walking new routes.

Because of the small sample size we consider our results an indication that the app can be of help and a good start for further research on guiding people through an urban environment with beacons.

\section{Acknowledgements}

This publication is made possible by the Amsterdam University of Applied Sciences grand Urban Vitality and Amsterdam Creative Industries Network.

\section{References}

1. James R. Marston and Reginald G. Golledge. 2003. The hidden demand for participation in activities and travel by persons who are visually impaired. Journal of Visual Impairment and Blindness, 97, 8, (August 2003), 475-488.

2. Jacqueline Broerse, Anne-Floor Schölvinck, Dirk Essink and Carina Pittens. 2015. Onderzoek Een onderzoeksagenda vanuit clientenperspectief. Retrieved July 23, 2016 from:

http://www.oogonderzoek.net/rapportzichtoponder zoek.pdf

3. John Morris and James Mueller. 2014. Blind and Deaf Consumer Preferences for Android and iOS Smartphones. Inclusive Designing (2014), 69-79. http://dx.doi.org/10.1007/978-3-319-05095-9_7

4. Nicholas A Giudice, Jonathan Z. Bakdash ad Gorden E. Legge. 2006. Wayfinding with words: Spatial learning and navigation using dynamically updated verbal descriptions. Psychological Research, 71,3 (2007), 347-358. http://dx.doi.org/10.1007/s00426-006-0089-8

5. Ross Atkin, Peter Buckle, and Jeremy Myerson. 2015. Street works and vision impairment: improving signing and guarding. Proceedings of the ICE - Municipal Engineer 168, 1, (January 2015) 11-23. http://dx.doi.org/10.1680/muen.14.00015

6. Else M. Havik, Aart C. Kooijman, Frank J.J. Steyvers. 2011. The Effectiveness of Verbal Information Provided by Electronic Persons. Journal of Visual Impairment \& Blindness, 105, 10 (October 2011), 624-638. 